\title{
Evidências de Validade para Instrumentos de Atenção e Funções Executivas e Relação com Desempenho Escolar
}

\author{
Gleize Urias da Silva da Fonseca \\ Ricardo Franco de Lima ${ }^{1}$ \\ Renata Eliane Ims \\ Danielle Ghirotti Coelho \\ Faculdade de Ciências Médicas da Universidade Estadual de Campinas, \\ Campinas, SP, Brasil \\ Sylvia Maria Ciasca \\ Departamento de Neurologia da Universidade Estadual de Campinas, Campinas, SP, Brasil
}

\begin{abstract}
Resumo
O trabalho objetivou investigar evidências de validade de instrumentos que avaliam atenção sustentada visual e componentes das Funções Executivas. Foram buscadas evidências com base no desenvolvimento, considerando efeitos da progressão do nível escolar e evidências baseadas na relação com variáveis externas, no caso com medida do desempenho escolar. Participaram 151 crianças, ambos os sexos (77 feminino e 74 masculino), de 7 a 11 anos $(M=8,6 ; D P=1,15)$, do $2^{\circ}$ ao $5^{\circ}$ ano, sem queixas de dificuldades de aprendizagem. Para a coleta dos dados foram utilizados os instrumentos Teste de Desempenho Escolar (TDE), Testes de Cancelamento, Teste das Trilhas, Teste Cor-Palavra de Stroop, Torre de Londres e Teste de Fluência Verbal. Os resultados revelaram melhora no desempenho com a progressão da escolaridade. Houve correlações significativas entre os escores dos instrumentos e o TDE, mesmo quando controlada a variável idade. Foram obtidas evidências de validade para os instrumentos, de modo que apresentam sensibilidade para detectar mudanças do desenvolvimento em função da progressão do ano escolar e relação com o desempenho em escrita, aritmética e leitura.
\end{abstract}

Palavras-chave: Neuropsicologia, avaliação, validade, atenção, funções executivas.

\section{Validity Evidences of Instruments of Attention and Executive Functions and their Relationship with School Performance}

\begin{abstract}
The study investigated validity evidences of instruments that assess visual sustained attention and executive functions components. We analyzed evidences-based developmental, considering effects of grade levels progression and evidence-based relationship with external variables, particularly school performance. Participated 151 children, both genders ( 77 female e 74 male), $7-11$ years old $(M=8.6 ; S D=$ 1.15 ), from the $2^{\text {nd }}$ to $5^{\text {th }}$ grade, no complaints from learning difficulties were evaluated by Academic Performance Test (TDE), Cancellation Tests, Trail Making Test, Stroop Color Word Test, Tower of
\end{abstract}

Endereço para correspondência: Universidade Estadual de Campinas, Departamento de Neurologia, Faculdade de Ciências Médicas, Laboratório de Pesquisa em Dificuldades, Distúrbios de Aprendizagem e Transtornos da Atenção, Caixa Postal 6111, Campinas, SP, Brasil 13083-970. E-mail: ricardolima01@yahoo.com.br Resultados do trabalho de conclusão de curso de Especialização em Neuropsicologia Aplicada à Neurologia Infantil. 
London and Verbal Fluency Test. The results showed improvement in performance with the progression of school degree. There were significant correlations between scores of the instruments and TDE, even when controlled for the age. This study provided evidences of validity for the instruments, since it was sensitive to detect changes due to the progression of the school degree and correlated with performance in writing, reading and arithmetic.

Keywords: Neuropsychology, assessment, validity, attention, executive functions.

\section{Evidencias de Validez para Instrumentos de Atención y Funciones Ejecutivas y su Relación com el Rendimiento Escolar}

\section{Resumen}

El estudio tuvo como objetivo investigar la evidencia de la validez de los instrumentos que evalúan la atención sostenida y los componentes visuales de las funciones ejecutivas. Desarrollo basado en la evidencia, teniendo en cuenta los efectos de la progresión del nivel de grado y la relación basada en la evidencia con las variables externas, como en el caso con el rendimiento escolar se buscaron. Participaron 151 niños de ambos sexos (77 niñas y 74 ninõs), 7-11 años de edad $(M=8.6 ; S D=1.15)$, desde el 2 al 5 de años, no tengo ninguna queja de las dificultades de aprendizaje. Para la recolección de datos se utilizaron la prueba de rendimiento académico (TDE), Test de Cancelación, Trail Making Test, Stroop Color Word Teste, Torre de Londres y la prueba de fluidez verbal. Los resultados mostraron una mejora en el rendimiento con la progresión de la escolarización. Se encontraron correlaciones significativas entre las puntuaciones de los instrumentos y TDE, incluso cuando se controla por la variable edad. Pruebas de validez de los instrumentos que cuentan por lo que la sensibilidad para detectar cambios en el desarrollo después de la progresión del ciclo escolar y en comparación con el rendimiento en la escritura, se obtuvieron lectura y aritmética.

Palabras clave: Neuropsicología, evaluación, validez, atención, funciones ejecutivas.

As diferentes funções cognitivas investigadas na avaliação neuropsicológica são: atenção, percepção, memória, praxias, linguagem, raciocínio e Funções Executivas (FE). Dentre elas a atenção e as FE estão diretamente ligadas às habilidades escolares (Hamdan, Pereira, \& Riechi, 2011; Simão, Lima, Natalin, \& Ciasca, 2010).

A atenção é compreendida como a capacidade de direcionamento dos processos mentais, considerando os estímulos relevantes e ignorando os irrelevantes durante o desempenho das tarefas (Lima, Travaini, \& Ciasca, 2009). Do ponto de vista neuropsicológico e clínico, a atenção pode ser dividida em: seletiva (capacidade de discriminar estímulos relevantes e irrelevantes), sustentada (manter o foco atencional em determinado estímulo por um período de tempo), alternada (alternar foco entre diferentes estímulos) e dividida (dividir foco atencional para desempenho de duas tarefas simultaneamente;
Lezak, Howieson, \& Loring, 2004; Simão et al., 2010).

Por outro lado, as FE são definidas como conjunto de habilidades mentais que ajudam o indivíduo a realizar tarefas de forma independente e direcionada para metas (Lima, Azoni, \& Ciasca, 2011). Essas funções, quando integradas, capacitam o indivíduo a tomar decisões, avaliar e adequar seus comportamentos e estratégias, buscando a resolução para um problema (Menezes, Godoy, Teixeira, Carreiro, \& Seabra, 2012). Além disso, facilitam o gerenciamento das outras habilidades cognitivas (Malloy-Diniz, Paula, Loschiavo-Alvares, Fuentes, \& Leite, 2010).

Entre os componentes cognitivos que fazem parte das FE podemos destacar: flexibilidade cognitiva (capacidade para mudar o curso de ação alternando o foco atencional e estratégias utilizadas), controle inibitório (habilidade de pensar antes de agir, postergar ou inibir res- 
postas a partir da avaliação de diversos fatores), planejamento (capacidade de estabelecer a melhor maneira de alcançar um objetivo definido) e fluência (capacidade do indivíduo emitir uma variedade de respostas verbais dentro de uma estrutura de regras específicas; León, Rodrigues, Seabra, \& Dias, 2013; Lima, Tabaquim, \& Ciasca, 2010; Malloy-Diniz et al., 2010).

No que se refere ao desenvolvimento atencional, certos aspectos estão presentes desde o nascimento e se modificam em diferentes períodos. Entretanto, no que tange à precisão e velocidade em tarefas visuais e auditivas que avaliam a atenção seletiva e sustentada, observa-se rápido desenvolvimento até os 10 anos de idade, quando a partir de então, as mudanças tornam-se menos perceptíveis (Carreiro \& Teixeira, 2012; Hazin et al., 2012).

No desenvolvimento das FE, alguns dos seus componentes, como a flexibilidade cognitiva e planejamento, possuem maturação tardia em relação às demais funções cognitivas (Pereira, Seabra, Dias, Trevisan, \& Prado, 2011). Assim, seu desenvolvimento tem início no primeiro ano de vida, é intenso entre 6 e 8 anos, continua até o início da idade adulta, e se estabiliza até o envelhecimento, quando começa a declinar (Menezes et al., 2012).

É possível evidenciar aspectos do desenvolvimento destas funções em relação à escolaridade. Observa-se que há relação entre o desempenho de crianças em testes de atenção e seu desempenho escolar (Capovilla \& Dias, 2008). Da mesma maneira, estudos têm demonstrado que o desenvolvimento das FE influencia na aprendizagem, contribuindo para o desempenho acadêmico (Capovilla \& Dias, 2008; León et al., 2013).

Desta forma, dificuldades atencionais e de funcionamento executivo podem influenciar o aprendizado de habilidades escolares, como a leitura e escrita, bem como o desenvolvimento destas funções cognitivas demonstra ser preditivo para o desempenho em disciplinas de linguagem e de matemática (León et al., 2013; Simão et al., 2010). Assim, por influenciar diretamente a aprendizagem, é importante que essas funções sejam investigadas.
No Brasil, a área de avaliação neuropsicológica é crescente. Apesar disto, ainda são escassos os trabalhos que investigam o desenvolvimento dessas habilidades ao longo das séries, em crianças saudáveis e principalmente em relação ao desempenho escolar (Capovilla \& Dias, 2008).

Embora haja a necessidade de desenvolver pesquisas nesse campo, existem diferentes instrumentos utilizados no Brasil para avaliar as funções cognitivas de atenção e executivas. No caso da avaliação da atenção sustentada para materiais visuais podemos citar o Teste de Cancelamento (TAC; Capovilla \& Dias, 2008; Dias, 2009; Dias \& Seabra, 2012; Hazin et al., 2012; Pereira et al., 2011; Seabra \& Dias, 2010) e os Testes de Cancelamento - Figuras Geométricas e Letras em Fileira (Lima et al., 2009; Simão et al., 2010).

Para avaliar os diferentes componentes das FE, outros instrumentos também são citados com paradigmas semelhantes, porém com estímulos ou versões diferentes: (a) Teste das Trilhas para avaliar atenção sustentada visual (Parte A) e flexibilidade (Parte B; Dias \& Seabra, 2012, 2014; Lima et al., 2009; Simão et al., 2010); (b) Torre de Londres (ToL) para avaliar planejamento e raciocínio lógico (Dias \& Seabra, 2012; Lima et al., 2009; Simão et al., 2010); (c) Teste de Fluência Verbal (FAS) para avaliar a fluência verbal (Dias \& Seabra, 2014); (d) Teste Cor-Palavra de Stroop (SCWT) para avaliar o controle inibitório e atenção seletiva (Charchat-Fichman \& Oliveira, 2009; Dias \& Seabra, 2012; Duncan, 2006; Lima et al., 2009; Simão et al., 2010).

É importante que os instrumentos neuropsicológicos apresentem parâmetros psicométricos adequados, geralmente obtidos por meio de estudos de evidências de validade e fidedignidade. Obter evidências de validade refere-se a investigar a adequação da intepretação das respostas dos indivíduos a determinados instrumentos (Carvalho, 2012). A validade também pode ser interpretada como uma questão de julgamento aos escores dos instrumentos (Urbina, 2007). Algumas fontes de evidências de validade são: baseadas no conteúdo, na estrutura interna, na relação com variáveis externas, no processo de 
resposta e nas consequências da testagem (Carvalho, 2012; Urbina, 2007).

Diferentes estudos basileiros têm sido conduzidos com intuito de obter evidências de validade para instrumentos de atenção e FE. Capovilla e Dias (2008) realizaram um estudo de validade, no qual investigaram o desenvolvimento de habilidades atencionais em estudantes e a relação com o rendimento escolar. Participaram deste estudo 407 estudantes (6 a 15 anos), de $1^{\text {a }}$ a $5^{\text {a }}$ séries do ensino fundamental, avaliados coletivamente com o Teste Atenção por Cancelamento (TAC) e o Teste de Trilhas - partes A e B (TMT), além da média das notas de diferentes disciplinas. O resultado indicou aumento dos escores dos anos sucessivos entre a $2^{\mathrm{a}}$ e a $4^{\mathrm{a}}$ série. Para o TMT-A foi evidenciado aumento nos escores das $1^{\mathrm{a}} \mathrm{e} 3^{\mathrm{a}}$ séries e no TMT-B discriminou-se apenas a $4^{\text {a }}$ série dos demais.

Similarmente, Seabra e Dias (2010) investigaram evidências de validade para o TAC e o TMT-B em 255 estudantes sem deficiências (10 a 17 anos), cursando do $6^{\circ}$ ao $9^{\circ}$ ano do ensino fundamental de uma escola pública do interior de SP. Foram obtidos efeitos dos anos escolares sobre os escores, visto que no TAC-total houve um aumento dos escores no $9^{\circ}$ em relação ao $6^{\circ}$ ano, e a terceira parte do teste, que avalia a atenção alternada, foi a que melhor discriminou os anos escolares. O TMT-B, discriminou o $6^{\circ}$ e $7^{\circ}$ em relação ao $9^{\circ}$ ano. Análises de correlação entre os testes foram significativas, sugerindo que os instrumentos avaliam constructos distintos, porém, relacionados.

Hazin et al. (2012) buscou dados normativos para o TAC em 524 estudantes (7 a 16 anos) cursando o ensino fundamental nas redes pública e particular de Natal/RN. Os resultados revelaram efeitos das variáveis "nível de escolaridade", "tipo de escola" e "faixa etária" em relação ao desempenho dos estudantes no TAC. Os resultados demonstraram ainda um aumento significativo no desempenho dos estudantes em relação à velocidade e à precisão nas tarefas de seleção e sustentação, nas três primeiras séries do ensino fundamental e estabilização dos resultados nos três últimos anos, sugerindo o es- tabelecimento de um platô no desenvolvimento atencional.

Em outro estudo, Pereira et al. (2011) avaliaram 85 crianças (4 a 6 anos) de uma escola municipal de ensino infantil e fundamental da grande SP, com objetivo de investigar a relação entre o desempenho em uma tarefa de atenção seletiva e o desenvolvimento das crianças nessa faixa etária, utilizando o TAC. Resultados mostraram que os desempenhos das crianças aumentaram com a progressão da escolaridade. Além disto, houve correlação entre os escores do instrumento, denotando a consistência entre seus escores.

O estudo de Lima et al. (2009) buscou caracterizar o desempenho de crianças sem dificuldades de aprendizagem, cursando da $1^{\mathrm{a}}$ a $4^{\mathrm{a}}$ série (7 a 10 anos) nos instrumentos: Testes de Cancelamentos - Figuras Geométricas e Letras em Fileira (TC - FG e LF), Teste das Trilhas (Trail Making Test [TMT]) - Partes A e B, Teste Cor-Palavra de Stroop (Stroop Color Word Test [SCWT]) e Torre de Londres (Tower of London $[\mathrm{ToL}])$. Os resultados indicaram que, principalmente em relação aos escores de tempo, os instrumentos foram capazes de discriminar o escore dos estudantes entre as séries sucessivas, sugerindo que o desempenho nos testes poderia acompanhar o desenvolvimento da atenção e das FE. O estudo também correlacionou os escores dos instrumentos usados com o desempenho escolar, avaliado pelo Teste de Desempenho Escolar (TDE). Houve correlações significativas, sugerindo que quanto melhor o desempenho nos subtestes do TDE (aritmética, escrita e leitura), menor é o prejuízo nos instrumentos.

Simão et al. (2010) também utilizaram o TC, TMT-A/B, SCWT e ToL em estudantes (7 a 12 anos), com e sem queixas de dificuldades de atenção e aprendizagem. O estudo objetivou comparar o desempenho dos grupos nos instrumentos investigados. Os resultados evidenciaram diferenças significativas nos escores entre os grupos, sendo que o grupo com queixa apresentou escores de tempo e erros aumentados em relação ao outro grupo. Desse modo, os instrumentos foram sensíveis para diferenciar grupos 
com e sem dificuldades atencionais e de aprendizagem.

Outros estudos nacionais também utilizaram versões dos outros instrumentos empregados nessa pesquisa. Duncan (2006) avaliou 132 estudantes (12 a 14 anos), cursando $6^{\mathrm{a}}$ a $8^{\mathrm{a}}$ série do ensino fundamental, em Niterói/RJ, utilizando o teste de Stroop. Foram analisadas diferenças entre estudantes de escolas públicas e particulares, quanto ao sexo e idade. No que se refere à idade, houve diferenças somente nos escores de tempo entre estudantes de 13 e 14 anos. Charchat-Fichman e Oliveira (2009) também utilizaram o SCWT (versão Victoria) em amostra de 119 crianças (7-10 anos). Foram encontrados efeitos da idade, principalmente nos escores de tempo dos três cartões (cor, palavra e cor-palavra). Isto é, houve diminuição no tempo de resolução ao longo das idades. Além disto, as crianças menores foram mais suscetíveis à condição de interferência, conforme revelaram as análises de correlação. Os resultados são interpretados em termos de desenvolvimento, no qual há melhoras na velocidade de processamento, seletividade atencional e controle inibitório ao longo dos anos.

Dias e Seabra (2012) verificaram efeito da idade sobre o desempenho de 124 adolescentes (11-14 anos) de uma escola pública de SP em tarefas de memória operacional, no escore de interferência de tempo do Stroop computadorizado e na Torres de Londres (ToL; versão adaptada de Krikorian, Bartok, \& Gay, 1994). Dias e Seabra (2014), utilizaram o teste FAS para investigar as contribuições das diferentes FE, idade e sexo para o desempenho no teste. Assim, investigaram quais variáveis são preditoras para o desempenho no teste de Fluência Verbal computadorizado (FAS; versão fonológica) em dois grupos: 263 crianças (6-10 anos) e 150 adolescentes (1014 anos). No primeiro, o modelo de análise de regressão demonstrou que a idade, a memória operacional auditiva e a flexibilidade são melhores preditores para o desempenho do FAS. No segundo grupo, o sexo, a memória operacional auditiva, a flexibilidade e o controle inibitório foram os melhores preditores.
Considerando os estudos expostos, realizados com a população brasileira e utilizando instrumentos similares, o presente trabalho se propôs investigar evidências de validade de instrumentos para avaliação da atenção sustentada visual e componentes das FE (flexibilidade, controle inibitório, planejamento e fluência) a partir do desempenho de estudantes do Ensino Fundamental. Foram investigadas evidências: (a) na relação com critério externo - considerando possíveis efeitos da progressão do nível escolar sobre os escores; e (b) na relação com variável externa - medida padronizada de desempenho escolar (escrita, aritmética e leitura).

\section{Método}

\section{Participantes}

Foram avaliados 151 estudantes sem queixas escolares, sendo 74 (49\%) do sexo masculino e 77 (51\%) do sexo feminino, com faixa etária entre 7 a 11 anos e idade média de 8 anos 6 meses $(D P=1,15)$. Os estudantes cursavam do $2^{\circ}$ ao $5^{\circ}$ ano do ensino fundamental de duas escolas particulares e uma escola pública, de uma cidade do interior de São Paulo, sendo a amostra total composta por $38(25 \%)$ do $2^{\circ}$ ano, 32 (21\%) do $3^{\circ}$ ano, 39 (26\%) do $4^{\circ}$ ano e 42 (28\%) do $5^{\circ}$ ano (Tabela 1$)$.

Os critérios para a seleção dos participantes foram: (a) não apresentar queixas de dificuldades de aprendizagem, conforme relato e indicação dos professores; (b) apresentar desempenho cognitivo-intelectual dentro do esperado para a faixa etária, aferido pelo Desenho da Figura Humana (DFH-III; Wechsler, 2003); (c) apresentar desempenho escolar dentro do esperado para o nível de escolaridade, conforme resultados do Teste de Desempenho Escolar (TDE; Stein, 1994); (d) ausência de déficits sensoriais não corrigidos. Foram incluídos os estudantes cujos pais autorizaram a participação por meio da assinatura do Termo de Consentimento Livre e Esclarecido (TCLE). 
Tabela 1

Caracterização da Amostra em Função dos Anos Escolares

\begin{tabular}{|c|c|c|c|c|c|}
\hline \multirow{2}{*}{ Variáveis } & \multicolumn{4}{|c|}{ Anos escolares } & \multirow[b]{2}{*}{ Total } \\
\hline & $2^{\circ}$ ano & $3^{\circ}$ ano & $4^{\circ}$ ano & $5^{\circ}$ ano & \\
\hline \multicolumn{6}{|l|}{ Idade } \\
\hline$M(D P)$ & $7,11(0,31)$ & $8,22(0,42)$ & $9,13(0,73)$ & $9,79(0,47)$ & $8,61(1,15)$ \\
\hline \multicolumn{6}{|l|}{ Sexo } \\
\hline Masculino & $15(39 \%)$ & $16(50 \%)$ & $19(49 \%)$ & $24(57 \%)$ & $74(49 \%)$ \\
\hline Feminino & $23(61 \%)$ & $16(50 \%)$ & $20(51 \%)$ & $18(43 \%)$ & $77(51 \%)$ \\
\hline \multicolumn{6}{|l|}{ DFH-III } \\
\hline Média & $26(68 \%)$ & $22(69 \%)$ & $27(69 \%)$ & $39(94 \%)$ & $114(75 \%)$ \\
\hline Acima da média & $9(24 \%)$ & $6(19 \%)$ & $8(21 \%)$ & $1(2 \%)$ & $24(15 \%)$ \\
\hline Superior & $1(3 \%)$ & $4(12 \%)$ & $2(5 \%)$ & $1(2 \%)$ & $8(5 \%)$ \\
\hline Muito Superior & $2(5 \%)$ & 0 & $2(5 \%)$ & $1(2 \%)$ & $5(3 \%)$ \\
\hline \multicolumn{6}{|l|}{$\mathrm{TDE}$ - total } \\
\hline Médio & $34(89 \%)$ & $27(84 \%)$ & $37(95 \%)$ & $40(95 \%)$ & $138(91 \%)$ \\
\hline Superior & $4(11 \%)$ & $5(16 \%)$ & $2(5 \%)$ & $2(5 \%)$ & $13(9 \%)$ \\
\hline Total & $38(25 \%)$ & $32(21 \%)$ & $39(26 \%)$ & $42(28 \%)$ & $151(100 \%)$ \\
\hline
\end{tabular}

Nota. Legenda. M: média; DP: desvio padrão; DFH: Desenho da Figura Humana; TDE: Teste de Desempenho Escolar.

\section{Instrumentos}

Testes de Cancelamento (TC; Lima et al., 2009; Weintraub \& Mesulam, 1985). Avalia atenção sustentada visual, sendo composto por duas partes: (a) Figuras Geométricas (TC-FG): o estudante deveria marcar os círculos encontrados o mais rápido que conseguisse em uma folha com uma sequência randômica de figuras geométricas; (b) Letras em Fileira (TC-LF): marcar todas as letras "A" em uma folha com letras distribuídas de forma randômica. Critérios para avaliação: tempo (cronometrado durante o teste e expresso em segundos), erros por omissão (EO, número de estímulos-alvo não assinalados), erros por adição (EA, número de estímulos não alvo assinalados) e erros total (soma dos erros de omissão e adição).

Teste das Trilhas (TMT, Partes A e B; Lima et al., 2009; Reitan, 1992). A parte A avalia atenção sustentada visual e é composto por uma folha com círculos numerados de 1 a 25, distribuídos de forma aleatória, na qual o estudante deveria traçar uma linha ligando a sequência numérica. A Parte B avalia um dos componentes das FE, a flexibilidade mental. É composto por uma folha com círculos contendo números e letras. $\mathrm{O}$ estudante deveria traçar uma linha ligando os círculos alternadamente seguindo, respectivamente, as ordens numérica e alfabética. A avaliação do desempenho foi realizada em termos de: tempo de execução em segundos, número de erros de sequência (ES), número de erros de alternância (EA) e erros total (soma dos erros de alternância e sequência).

Teste Cor-Palavra de Stroop (SCWT; Lima et al., 2009; Regard, 1981). Avalia o controle inibitório e a capacidade do estudante em considerar estímulos relevantes e desconsiderar os estímulos não-relevantes. Foi utilizada uma adaptação da versão Victoria (Spreen \& Strauss, 1998) com quatro cores (vermelho, amarelo, azul e verde) e 24 estímulos em cada uma das três partes: (a) "Cartão Cores" (C) - nomear os quadrados pintados nas quatro cores organizadas 
em ordem randômica; (b) "Cartão Palavras" (P) - dizer os nomes das cores impressas, que agora estavam nas cores correspondentes; (c) "Cartão Cor-Palavra" (CP) - dizer o nome da cor e não ler a palavra. O desempenho foi medido pelo: tempo em segundos e erros (número de respostas incorretas) para cada cartão.

Torre de Londres (ToL; Lima et al., 2009; Tunstall, 1999). Avalia a habilidade de planejamento e de raciocínio lógico. Composta por uma base com três pinos verticais equidistantes e quatro discos coloridos do mesmo tamanho, com um furo no centro para possibilitar o encaixe no pino. A tarefa consistia em mover os discos coloridos para reproduzir, em um número determinado de movimentos, a posição mostrada em um caderno de estímulos. Existiam 10 itens com posições alvo com grau crescente de dificuldade, nos quais para chegar a uma posição final, o estudante deveria mover os discos, saindo de uma posição inicial colocada pelo experimentador, seguindo regras da quantidade de movimentos. Considerou-se como movimento a retirada do círculo do pino e a colocação em outro. Foram permitidas três tentativas para a resolução de cada item. A resposta era considerada correta quando a solução era alcançada com o número exigido de movimentos. As respostas poderiam variar de 1, 2, 3 pontos, conforme tinham sido atingidas na terceira, segunda ou primeira tentativas, respectivamente. Foi considerado o escore total obtido pelo somatório dos escores de todos os itens completados. Desta maneira, quanto maior o escore, melhor o desempenho.

Teste de Fluência Verbal (FAS; Benton \& Hamsher, 1989). Avalia a fluência verbal, isto é, habilidade de produção de palavras verbalmente, iniciação verbal, organização e acesso ao léxico. Foram utilizadas duas partes do FAS: (a) Fonológica (FAS-F) - dizer o maior número de palavras que começassem com as letras "F", "A" e "S" em um minuto cada; (b) Semântica (FAS-S) - dizer o maior número de palavras nas categorias "animais", "frutas" e "materiais escolares" em um minuto cada. Os escores foram considerados em termos da soma de itens expressos em cada uma das partes.
Teste de Desempenho Escolar (TDE; Stein, 1994). Trata-se de um instrumento padronizado para a população brasileira e que visa oferecer uma avaliação das capacidades fundamentais para o desempenho escolar: leitura, escrita e matemática. Tal teste foi concebido a partir da avaliação do desempenho de escolares cursando o $2^{\circ}$ ao $7^{\circ}$ ano do ensino fundamental, ainda que possa ser aplicado com algumas reservas para a $8^{\circ}$ e $9^{\circ}$ ano. O TDE é formado por três subtestes: (a) Escrita; (b) Leitura e; (c) Aritmética. Cada um apresenta uma escala de itens em ordem crescente de dificuldade. Os acertos são pontuados, definindo o Escore Bruto (EB) de cada um dos subtestes. Tais dados são convertidos por meio de uma tabela, conforme o nível escolar e são obtidas as classificações em níveis: superior, médio e inferior. Para o presente estudo, foram consideradas as classificações dos estudantes a partir do escore total e os escores brutos por subtestes para realizar correlações com os instrumentos de atenção e FE.

\section{Procedimentos}

Após aprovação pelo Comitê de Ética (Protocolo $\mathrm{n}^{\circ} 483.860$ ), foram realizados contatos com escolas do ensino fundamental, com objetivo de obter os consentimentos para realização da pesquisa mediante uma carta de apresentação e autorização. Além disso, foi realizada reunião com os professores para a apresentação da pesquisa, e solicitado que indicassem os estudantes de acordo com os critérios de inclusão e exclusão. Em seguida, foi marcada uma reunião com os pais dos estudantes para explicação sobre o projeto e seus aspectos éticos, bem como a assinatura do TCLE. Posteriormente, foram iniciados os encontros com os estudantes para a administração dos instrumentos. As avaliações foram realizadas de forma individual, em uma sessão com duração de 60 minutos em média, em uma sala determinada pela escola. Após o término das avaliações foram marcadas sessões de devolutiva com os pais, entregue relatório com os resultados e agendada nova reunião com a equipe escolar para devolutiva do trabalho realizado. 


\section{Análise dos Dados}

Para a análise dos dados foi utilizada a estatística descritiva e inferencial por meio da planilha do Programa SPSS versão 20.0. Para cada instrumento utilizado foram realizadas análises descritivas na amostra total e em função do nível de escolaridade. Considerando os resultados das análises de distribuição e homogeneidade dos dados (Kolmogorov-Smirnov) optou-se pelo uso de testes não paramétricos. A análise do efeito do ano escolar foi realizada por meio do teste de Kruskall-Wallis, complementado pelo teste de Mann-Whitney. Posteriormente foi conduzi-

Tabela 2

Estatística Descritiva dos Escores dos Instrumentos utilizados na Amostra Total

\begin{tabular}{lcccc}
\hline \multicolumn{1}{c}{ Escores } & $M$ & $D P$ & Mínimo & Máximo \\
\hline TC-FG tempo & 87,06 & 20,86 & 50 & 175 \\
TC-FG_EO & 1,14 & 2,49 & 0 & 16 \\
TC-FG_EA & 0,00 & 0,00 & 0 & 0 \\
TC-FG erros total & 1,14 & 2,49 & 0 & 16 \\
TC-LF tempo & 135,80 & 44,84 & 76 & 358 \\
TC-LF EO & 4,17 & 5,59 & 0 & 31 \\
TC-LF EA & 0,01 & 0,08 & 0 & 1 \\
TC-LF erros total & 4,18 & 5,59 & 0 & 31 \\
TMT-A tempo & 48,88 & 17,56 & 19 & 107 \\
TMT-A erros & 0,19 & 0,62 & 0 & 4 \\
TMT-B tempo & 118,28 & 43,67 & 53 & 240 \\
TMT-B_EA & 0,21 & 0,67 & 0 & 4 \\
TMT-B_ES & 0,18 & 0,60 & 0 & 4 \\
TMT-B total erros & 0,39 & 1,08 & 0 & 6 \\
SCWT-C tempo & 19,28 & 5,18 & 11 & 42 \\
SCWT-C erros & 0,13 & 0,41 & 0 & 2 \\
SCWT-P tempo & 14,94 & 4,68 & 8 & 38 \\
SCWT-P erros & 0,05 & 0,32 & 0 & 3 \\
SCWT-CP tempo & 36,62 & 10,53 & 19 & 85 \\
SCWT-CP erros & 1,64 & 1,86 & 0 & 87 \\
FAS fonológico & 22,32 & 6,47 & 9 & 38 \\
FAS semântico & 34,16 & 6,67 & 19 & 8 \\
ToL & 18,83 & 3,71 & 07 & 3 \\
\hline
\end{tabular}

Nota. Legenda. TC-FG_EO - Teste de Cancelamento - Figuras Geométricas erros de omissão; TC-FG_EA - erros de adição; TC-LF EO - Teste de Cancelamento - Letras em Fileira erros de omissão; TC-LF EA - erros de adição; TMT-A - Teste das Trilhas A; TMT-B_EA - Teste das Trilhas B erros de alternância; TMT-B_ES - erros de sequência; SCWT - C - Teste Stroop Cor; SCWT - P - Teste Stroop Palavra; SCWT - CP - Teste Stroop Cor-Palavra; FAS - Fluência Verbal; ToL - Torre de Londres. 
$\mathrm{Na}$ análise em função dos anos escolares (Tabela 3), foi possível observar que no TC-FG e TC-LF houve diminuição sistemática de tempo e de erros por omissão com a evolução dos anos escolares. Nota-se um aumento das médias de tempo dos instrumentos TC-FG, TC-LF, TMT parte A e $\mathrm{B}$ no $5^{\circ}$ ano quando comparado com o $4^{\circ}$ ano. Ainda, no teste de TMT- B foi encontrado um aumento de erros (Total, alternância e sequência) nos escores dos estudantes do $3^{\circ}$ ano em relação

Tabela 3

Estatística Descritiva dos Escores dos Instrumentos de Atenção e Funções Executivas em Função dos Anos Escolares

\begin{tabular}{|c|c|c|c|c|c|c|}
\hline Escores & Ano & $N$ & $M$ & $D P$ & Mínimo & Máximo \\
\hline \multirow[t]{4}{*}{ TC-FG tempo } & 2 & 38 & 94,32 & 16,61 & 69 & 138 \\
\hline & 3 & 32 & 93,53 & 23,98 & 58 & 170 \\
\hline & 4 & 39 & 80,90 & 22,08 & 52 & 175 \\
\hline & 5 & 42 & 81,29 & 17,45 & 50 & 127 \\
\hline \multirow[t]{4}{*}{ TC-FG_EO } & 2 & 38 & 1,97 & 3,54 & 0 & 15 \\
\hline & 3 & 32 & 1,47 & 3,12 & 0 & 16 \\
\hline & 4 & 39 & 0,77 & 1,39 & 0 & 5 \\
\hline & 5 & 42 & 0,48 & 0,97 & 0 & 4 \\
\hline \multirow[t]{4}{*}{ TC-LF tempo } & 2 & 38 & 157,61 & 44,44 & 77 & 302 \\
\hline & 3 & 32 & 141,31 & 47,15 & 92 & 358 \\
\hline & 4 & 39 & 122,10 & 29,78 & 82 & 212 \\
\hline & 5 & 42 & 124,60 & 47,95 & 76 & 311 \\
\hline \multirow[t]{4}{*}{ TC-LF_EO } & 2 & 38 & 6,79 & 7,55 & 0 & 31 \\
\hline & 3 & 32 & 4,72 & 5,49 & 0 & 21 \\
\hline & 4 & 39 & 3,72 & 4,52 & 0 & 18 \\
\hline & 5 & 42 & 1,81 & 2,92 & 0 & 12 \\
\hline \multirow[t]{4}{*}{ TMT-A tempo } & 2 & 38 & 53,82 & 16,37 & 25 & 88 \\
\hline & 3 & 32 & 47,59 & 13,79 & 26 & 94 \\
\hline & 4 & 39 & 43,33 & 16,64 & 21 & 107 \\
\hline & 5 & 42 & 50,55 & 20,74 & 19 & 96 \\
\hline \multirow[t]{4}{*}{ TMT-A erros } & 2 & 38 & 0,26 & 0,64 & 0 & 3 \\
\hline & 3 & 32 & 0,50 & 1,05 & 0 & 4 \\
\hline & 4 & 39 & 0,05 & 0,22 & 0 & 1 \\
\hline & 5 & 42 & 0,00 & 0,00 & 0 & 0 \\
\hline \multirow[t]{4}{*}{ TMT-B tempo } & 2 & 38 & 134,05 & 45,20 & 61 & 226 \\
\hline & 3 & 32 & 129,72 & 39,14 & 85 & 240 \\
\hline & 4 & 39 & 104,00 & 40,54 & 53 & 200 \\
\hline & 5 & 42 & 108,55 & 42,77 & 54 & 193 \\
\hline \multirow[t]{4}{*}{ TMT-B_EA } & 2 & 38 & 0,18 & 0,69 & 0 & 4 \\
\hline & 3 & 32 & 0,53 & 1,05 & 0 & 4 \\
\hline & 4 & 39 & 0,10 & 0,38 & 0 & 2 \\
\hline & 5 & 42 & 0,10 & 0,37 & 0 & 2 \\
\hline
\end{tabular}

aos estudantes do $2^{\circ}$ ano. Observou-se no SCWT que todos os escores apresentaram escores de tempo e erro menores com a evolução dos anos escolares, exceto nos escores SCWT-C e SCWT-CP erros, nos quais houve aumento na média do $3^{\circ}$ ano quando comparado com o $2^{\circ}$ ano, e do $5^{\circ}$ com o $4^{\circ}$ ano, respectivamente. Os escores do teste FAS demonstraram aumento do número de palavras nas duas categorias com a progressão dos anos escolares. Da mesma maneira, houve número maior de acertos nos escores da ToL. 


\begin{tabular}{|c|c|c|c|c|c|c|}
\hline Escores & Ano & $N$ & $M$ & $D P$ & Mínimo & Máximo \\
\hline \multirow[t]{4}{*}{ TMT-B_ES } & 2 & 38 & 0,05 & 0,23 & 0 & 1 \\
\hline & 3 & 32 & 0,53 & 1,08 & 0 & 4 \\
\hline & 4 & 39 & 0,10 & 0,38 & 0 & 2 \\
\hline & 5 & 42 & 0,10 & 0,37 & 0 & 2 \\
\hline \multirow[t]{4}{*}{ TMT-B total erros } & 2 & 38 & 0,24 & 0,79 & 0 & 4 \\
\hline & 3 & 32 & 1,06 & 1,78 & 0 & 6 \\
\hline & 4 & 39 & 0,21 & 0,77 & 0 & 4 \\
\hline & 5 & 42 & 0,19 & 0,59 & 0 & 2 \\
\hline \multirow[t]{4}{*}{ SCWT-C tempo } & 2 & 38 & 21,74 & 4,98 & 16 & 42 \\
\hline & 3 & 32 & 21,69 & 6,37 & 14 & 40 \\
\hline & 4 & 39 & 18,36 & 3,62 & 13 & 29 \\
\hline & 5 & 42 & 16,07 & 3,40 & 11 & 30 \\
\hline \multirow[t]{4}{*}{ SCWT-C erros } & 2 & 38 & 0,13 & 0,47 & 0 & 2 \\
\hline & 3 & 32 & 0,16 & 0,37 & 0 & 1 \\
\hline & 4 & 39 & 0,13 & 0,34 & 0 & 1 \\
\hline & 5 & 42 & 0,12 & 0,45 & 0 & 2 \\
\hline \multirow[t]{4}{*}{ SCWT-P tempo } & 2 & 38 & 17,61 & 4,96 & 11 & 31 \\
\hline & 3 & 32 & 17,12 & 5,20 & 11 & 38 \\
\hline & 4 & 39 & 13,69 & 2,97 & 9 & 24 \\
\hline & 5 & 42 & 12,02 & 2,98 & 8 & 20 \\
\hline \multirow[t]{4}{*}{ SCWT-P erros } & 2 & 38 & 0,13 & 0,41 & 0 & 2 \\
\hline & 3 & 32 & 0,09 & 0,53 & 0 & 3 \\
\hline & 4 & 39 & 0,00 & 0,00 & 0 & 0 \\
\hline & 5 & 42 & 0,00 & 0,00 & 0 & 0 \\
\hline \multirow[t]{4}{*}{ SCWT-CP tempo } & 2 & 38 & 44,16 & 12,96 & 25 & 85 \\
\hline & 3 & 32 & 37,91 & 9,49 & 20 & 58 \\
\hline & 4 & 39 & 33,64 & 7,10 & 22 & 48 \\
\hline & 5 & 42 & 31,60 & 7,17 & 19 & 47 \\
\hline \multirow[t]{4}{*}{ SCWT-CP erros } & 2 & 38 & 1,84 & 1,75 & 0 & 6 \\
\hline & 3 & 32 & 1,47 & 2,00 & 0 & 8 \\
\hline & 4 & 39 & 1,44 & 1,89 & 0 & 8 \\
\hline & 5 & 42 & 1,76 & 1,87 & 0 & 6 \\
\hline \multirow[t]{4}{*}{ FAS fonológico } & 2 & 30 & 18,87 & 4,85 & 11 & 30 \\
\hline & 3 & 23 & 20,78 & 6,36 & 9 & 34 \\
\hline & 4 & 30 & 22,83 & 5,53 & 11 & 34 \\
\hline & 5 & 32 & 26,19 & 6,76 & 13 & 38 \\
\hline \multirow[t]{4}{*}{ FAS semântico } & 2 & 30 & 30,20 & 5,08 & 19 & 39 \\
\hline & 3 & 23 & 34,04 & 7,48 & 21 & 47 \\
\hline & 4 & 30 & 34,47 & 6,87 & 23 & 47 \\
\hline & 5 & 32 & 37,66 & 5,25 & 30 & 50 \\
\hline \multirow[t]{4}{*}{ ToL } & 2 & 38 & 17,47 & 3,20 & 12 & 27 \\
\hline & 3 & 32 & 17,91 & 4,27 & 8 & 26 \\
\hline & 4 & 39 & 19,38 & 3,98 & 11 & 27 \\
\hline & 5 & 42 & 20,24 & 2,83 & 12 & 24 \\
\hline
\end{tabular}

Nota. Legenda. TC-FG EO - Teste de Cancelamento - Figuras Geométricas erros de omissão; TC-FG EA - erros de adição; TC-LF_EO - Teste de Cancelamento - Letras em Fileira erros de omissão; TC-LF_EA - erros de adição; TMT-A - Teste das Trilhas A; TMT-B EA - Teste das Trilhas B erros de alternância; TMT-B ES - erros de sequência; SCWT - C - Teste Stroop Cor; SCWT - P - Teste Stroop Palavra; SCWT - CP - Teste Stroop Cor-Palavra; FAS - Fluência Verbal; ToL - Torre de Londres; $N$ : número; $M$ : média; $D P$ : desvio padrão. 
A análise do efeito do ano escolar sobre o desempenho nos instrumentos pode ser visualizada na Tabela 4. Houve diferenças estatisti- camente significativas entre os anos, principalmente nos escores de tempo, com exceção dos escores TC-FG erros por omissão, SCWT-C e SCWT-CP erros.

Tabela 4

Resultados da Comparação das Médias obtidas pelos Testes Kruskall-Wallis e Mann-Whitney em Função do Ano Escolar

\begin{tabular}{lcc}
\hline Escores & Valor de $p^{\mathrm{a}}$ & Diferenças entre anos escolares $^{\mathrm{b}}$ \\
\hline TC-FG tempo & $0,001^{*}$ & $2>4 ; 2>5 ; 3>5$ \\
TC-FG_EO & 0,078 & $2>4 ; 2>5 ; 3>5$ \\
TC-LF tempo & $0,001^{*}$ & $2>4 ; 3>5$ \\
TC-LF_EO & $0,001^{*}$ & $2>4 ; 2>5 ; 3>5$ \\
TMT-A tempo & $0,008^{*}$ & $2>4$ \\
TMT-A erros & $0,004^{*}$ & $2>5 ; 3>4 ; 3>5$ \\
TMT-B tempo & $0,001^{*}$ & $2>4 ; 2>5 ; 3>4$ \\
TMT-B_EA & $0,024^{*}$ & $3>2 ; 3>5$ \\
TMT-B_ES & $0,021^{*}$ & $3>2 ; 3>5$ \\
TMT-B total erros & $0,004^{*}$ & $3>2 ; 3>5$ \\
SCWT-C tempo & $0,001^{*}$ & $2>5$ \\
SCWT-C erros & 0,680 & $3>5$ \\
SCWT-P tempo & $0,001^{*}$ & $2>5 ; 3>5$ \\
SCWT-P erros & $0,032^{*}$ & $2>5 ; 3>5$ \\
SCWT-CP tempo & $0,001^{*}$ & $2>5 ; 3>5$ \\
SCWT-CP erros & 0,422 & $2>4$ \\
FAS fonológico & $0,001^{*}$ & $4>2 ; 5>2$ \\
FAS semântico & $0,001^{*}$ & $4>2 ; 5>2$ \\
ToL & $0,001^{*}$ & $4>2 ; 5>2$ \\
\hline
\end{tabular}

Notas. Legenda. TC-FG_EO - Teste de Cancelamento - Figuras Geométricas erros de omissão; TC-FG_EA - erros de adição; TC-LF EO - Teste de Cancelamento - Letras em Fileira erros de omissão; TC-LF EA - erros de adição; TMT-A - Teste das Trilhas A; TMT-B_EA - Teste das Trilhas B erros de alternância; TMT-B_ES - erros de sequência; SCWT - C - Teste Stroop Cor; SCWT - P - Teste Stroop Palavra; SCWT - CP - Teste Stroop Cor-Palavra; FAS - Fluência Verbal; ToL - Torre de Londres; Esc. - Escrita; Arit. - Aritmética; Leit. - Leitura. *Valor significativo.

${ }^{\mathrm{a}}$ Kruskall-Wallis; ${ }^{\mathrm{b}}$ Mann-Whitney.

Foram conduzidas análises de correlação de Spearman entre os instrumentos e o TDE e, posteriormente, a análise de correlação parcial controlando efeito da idade (Tabela 5). Foram obtidas correlações significativas entre diferentes escores dos instrumentos em relação aos subtestes do TDE (escrita, aritmética e leitura), com magnitudes que variaram entre baixas e modera- das. Correlações significativas e positivas ocorreram nos escores do FAS nas duas categorias e do ToL. Já as significativas e negativas ocorreram na maior parte dos escores, principalmente no que se refere ao tempo. Na correlação parcial controlando a variável idade, de modo geral, identificou-se diminuição de correlações significativas. 
Tabela 5

Matriz de Correlação de Spearman entre os Instrumentos de Avaliação da Atenção e das Funções Executivas e o Teste de Desempenho Escolar (TDE)

\begin{tabular}{llllllc}
\hline \multicolumn{1}{c}{ Escores e TDE } & \multicolumn{2}{c}{ Coeficiente de Correlação de Spearman } & \multicolumn{2}{c}{$\begin{array}{c}\text { Coeficiente de correlação parcial } \\
\text { controlando variável 'idade' }\end{array}$} \\
\hline & Esc. & Arit. & Leit. & Esc. & Arit. & Leit. \\
\hline TC-FG tempo & $-0,28^{* *}$ & $-0,36^{* *}$ & $-0,22^{*}$ & $-0,05$ & $-0,06$ & $-0,03$ \\
TC-FG_EO & $-0,29^{* *}$ & $-0,31^{* *}$ & $-0,24^{*}$ & $-0,30^{*}$ & $-0,33^{* *}$ & $-0,24^{*}$ \\
TC-LF tempo & $-0,33^{* *}$ & $-0,31^{* *}$ & $-0,16$ & 0,01 & 0,05 & 0,10 \\
TC-LF_EO & $-0,36^{* *}$ & $-0,43^{* *}$ & $-0,29^{* *}$ & $-0,37^{* *}$ & $-0,45^{* *}$ & $-0,39^{* *}$ \\
TMT-A tempo & $-0,26^{* *}$ & $-0,42^{* *}$ & $-0,16$ & $-0,08$ & $-0,25^{*}$ & 0,03 \\
TMT-A erros & $-0,10$ & $-0,17$ & $-0,12$ & 0,06 & $0,01^{*}$ & 0,12 \\
TMT-B tempo & $-0,33^{* *}$ & $-0,46^{* *}$ & $-0,28^{* *}$ & $-0,16$ & $-0,25$ & 0,05 \\
TMT-B total & 0,03 & $-0,07$ & $-0,02$ & $-0,01$ & $-0,12$ & $-0,01$ \\
SCWT-C tempo & $-0,38^{* *}$ & $-0,39^{* *}$ & $-0,30^{* *}$ & $-0,27^{*}$ & $-0,44^{* *}$ & $-0,31^{*}$ \\
SCWT-C erros & $-0,17$ & $-0,18$ & $-0,15$ & $-0,21$ & $-0,29^{*}$ & $-0,08$ \\
SCWT-P tempo & $-0,40^{* *}$ & $-0,35^{* *}$ & $-0,32^{* *}$ & $-0,35^{* *}$ & $-0,32^{*}$ & $-0,32^{*}$ \\
SCWT-P erros & $-0,29^{* *}$ & $-0,18$ & $-0,30^{* *}$ & $-0,23$ & $-0,13$ & $-0,12$ \\
SCWT-CP tempo & $-0,51^{* *}$ & $-0,48^{* *}$ & $-0,43^{* *}$ & $-0,27^{*}$ & $-0,41^{* *}$ & $-0,20$ \\
SCWT-CP erros & $-0,35^{* *}$ & $-0,34^{* *}$ & $-0,32^{* *}$ & $-0,21$ & $-0,38^{* *}$ & $-0,03$ \\
FAS fonológico & $0,34^{* *}$ & $0,26^{*}$ & $0,44^{* *}$ & $0,31^{*}$ & 0,09 & $0,32^{*}$ \\
FAS semântico & $0,34^{* *}$ & $0,37^{* *}$ & $0,36^{* *}$ & 0,25 & 0,19 & 0,27 \\
ToL & 0,15 & $0,22^{*}$ & $0,20^{*}$ & 0,18 & 0,21 & 0,06 \\
\hline
\end{tabular}

Notas. Legenda. TC-FG_EO - Teste de Cancelamento - Figuras Geométricas erros de omissão; TC-FG_EA - erros de adição; TC-LF_EO - Teste de Cancelamento - Letras em Fileira erros de omissão; TC-LF_EA - erros de adição; TMT-A - Teste das Trilhas A; TMT-B_EA - Teste das Trilhas B erros de alternância; TMT-B_ES - erros de sequência; SCWT - C - Teste Stroop Cor; SCWT - P - Teste Stroop Palavra; SCWT - CP - Teste Stroop Cor-Palavra; FAS - Fluência Verbal; ToL - Torre de Londres; Esc. - Escrita; Arit. - Aritmética; Leit. - Leitura.

${ }^{*} p<0,05$; ${ }^{*} p<0,01$.

\section{Discussão}

O presente estudo objetivou investigar evidências de validade de instrumentos que avaliam atenção sustentada visual e componentes das FE a partir do desempenho de estudantes sem dificuldades de aprendizagem, que cursavam do $2^{\circ}$ ao $5^{\circ}$ ano do ensino fundamental.

Inicialmente foram investigadas evidências na relação com critério externo, considerando possíveis efeitos da progressão do nível escolar. Nas análises descritivas e inferenciais, observou-se que os escores diferiram significa- tivamente os anos escolares em todos os instrumentos. Foi possível identificar diminuição sistemática do tempo e erros por omissão com a evolução dos anos escolares, como pode ser observado no TC-FG e no TC-LF. Estudos prévios com a mesma (Lima et al., 2009) e com outra versão do Teste de Cancelamento (TAC) também obtiveram resultados similares, indicando melhora no desempenho com a progressão dos anos escolares (Capovilla \& Dias, 2008; Pereira et al., 2011; Seabra \& Dias, 2010). Esses resultados sugerem que com o avanço da escolaridade e o aumento da demanda atencional, as 
crianças tendem a ser mais rápidas no teste de atenção sustentada.

Nota-se aumento discreto nos escores de tempo do TC-FG, TC-LF, TMT parte A e B no $5^{\circ}$ quando comparado com $4^{\circ}$ ano, e no TMT-B, aumento de erros (Total, alternância e sequência) nos escores dos estudantes do $3^{\circ}$ em relação ao $2^{\circ}$ ano. Embora tal diferença seja pouco expressiva, os resultados gerais desses escores evidenciam que ainda houve tendência à diminuição dos escores (tempo/erros), o que pode ser inferido a partir da comparação entre os anos escolares iniciais e finais. Assim, os resultados ilustram que a amostra destes anos escolares, para estes escores, tendem a ser mais homogêneas, com escores médios próximos. Esses resultados corroboram com o estudo de Seabra e Dias (2010).

No SWCT, todos os escores apresentaram escores de tempo e erros menores com a evolução dos anos escolares, exceto os escores de erros dos Cartões SCWT-C e SCWT-CP que não obtiveram diferenças significativas, bem como houve um aumento sutil do escore do $3^{\circ}$ ano, quando comparado com o $2^{\circ}$ ano, e do $5^{\circ} \mathrm{com}$ parado com $4^{\circ}$ ano. Apesar das diferenças entre esses anos escolares, verifica-se que nos pontos extremos de escolaridade $\left(2^{\circ}\right.$ e $5^{\circ}$ anos $)$ houve evolução do desempenho no decorrer dos anos sucessivos.

Tais resultados corroboram com outros estudos que indicaram que com avanço escolar as crianças diminuem o tempo de execução, isto é, são mais rápidas para nomear as cores e aumentam o controle da interferência (Charchat-Fichman \& Oliveira, 2009; Lima et al., 2009; Simão et al., 2010).

Também foi encontrado efeito dos anos escolares sobre o desempenho no FAS e na ToL, com aumento sistemático nos escores. Os resultados podem sugerir que há relações entre o aumento das demandas ocasionadas pela escolaridade sobre o desenvolvimento e o desempenho em fluência verbal, planejamento e raciocínio lógico, aferidos pelo FAS e pela ToL. Outros estudos utilizando tais instrumentos, também encontraram efeitos da idade e escolaridade (Dias \& Seabra, 2012, 2014; León et al., 2013).
Em síntese, os resultados sugerem que particularmente os escores de tempo (TC, TMT, SCWT) e acertos (FAS e ToL) foram capazes de discriminar o desempenho dos participantes entre anos escolares e sua relação com o desenvolvimento da atenção e FE.

Como segundo objetivo do trabalho, foram investigadas evidências de validade dos instrumentos baseadas em suas relações com variáveis externas, no caso, o desempenho escolar. Para isto utilizamos um instrumento padronizado para a população brasileira o qual avalia o desempenho escolar em escrita, aritmética e leitura. Convém ressaltar que todos os estudantes que fizeram parte do estudo obtiveram resultados satisfatórios no TDE, com desempenhos classificados como médio ou superior.

No geral, foram obtidas correlações significativas entre os escores dos instrumentos de atenção e FE e os subtestes do TDE, com magnitudes que variaram entre baixas e moderadas. Foram encontradas correlações negativas e significativas, principalmente entre os escores de tempo (TC, TMT-B, SCWT) e o desempenho em escrita, aritmética e leitura. Tais resultados sugerem que pode existir algum tipo de associação entre o desempenho atencional, no que se refere ao tempo de execução, e o desempenho escolar. Esta interpretação possui respaldo teórico e clínico uma vez que o desempenho escolar depende da eficiência no controle atencional, representada pelos escores de tempo dos instrumentos (Capovilla \& Dias, 2008; León et al., 2013; Lima et al., 2009). Resultados semelhantes foram encontrados em estudo prévio com as mesmas versões dos instrumentos aqui investigadas (Lima et al., 2009).

Adicionalmente, correlações significativas e positivas foram obtidas entre os escores das duas categorias do FAS e os três subtestes do TDE, e entre o escore da ToL e subtestes Aritmética e Leitura. As correlações positivas entre estes escores podem ser justificadas teoricamente, considerando que o FAS também é um instrumento que avalia a capacidade de evocação de palavras (Benton \& Hamsher, 1989) e a ToL, o raciocínio lógico (Lima et al., 2009; Tunstall, 1999). Algumas destas relações podem ser menos esperadas, 
do ponto de vista teórico, como por exemplo, ToL e subteste de Leitura, os quais envolvem construtos diferentes. No entanto, outros estudos são necessários para investigar a participação destes componentes das FE e as habilidades escolares.

A fim de investigarmos possíveis influências da variável idade sobre as correlações obtidas entre os instrumentos e o TDE, conduzimos nova análise de correlação. De forma geral, houve diminuição na magnitude das correlações. No entanto, foram mantidas as correlações negativas e significativas, principalmente entre o TDE e os escores de erros dos TC e com os escores de erros dos cartões do SCWT. No FAS foram mantidas correlações somente entre a categoria fonológica e os subtestes de Escrita e Leitura do TDE. Tais achados sugerem que a variável idade pode interferir sobre o desempenho dos instrumentos, visto que a atenção e as FE seguem trajetórias que acompanham o desenvolvimento dos indivíduos (Carreiro \& Teixeira, 2012; Hazin et al., 2012; Menezes et al., 2012; Pereira et al., 2011).

Esta hipótese explicativa é coerente com dados da literatura que mostram o efeito da idade sobre o desempenho de estudantes em instrumentos de atenção (Hazin et al., 2012) e FE (Charchat-Fichman \& Oliveira, 2009; Dias \& Seabra, 2012; Duncan, 2006).

Em suma, foram verificadas correlações entre os escores dos instrumentos, principalmente referentes ao tempo e acertos, e o desempenho escolar aferido pelo TDE. No entanto, tais correlações podem ser influenciadas por efeito da idade.

\section{Conclusão}

Os achados do presente estudo nos permitem concluir que foram obtidas evidências de validade para as versões dos instrumentos de atenção e FE (Testes de Cancelamento, Teste das Trilhas, Teste Cor-Palavra de Stroop, Torre de Londres e Teste de Fluência Verbal) em estudantes do ensino fundamental, sem dificuldades de aprendizagem. Os resultados revelaram que os instrumentos foram sensíveis para detectar mudanças no desenvolvimento em função da progressão do ano escolar e relação com o desempenho em escrita, aritmética e leitura. Como limitações do estudo, podemos indicar a necessidade de controle mais rigoroso de algumas variáveis, tais como o desempenho cognitivo que, neste estudo, foi utilizado somente como critério para seleção dos participantes. Além disto os resultados tratam-se de dois tipos de evidência de validade (relação com critério e variável externa). Destacamos que os trabalhos seguintes devem complementar e investigar outros tipos de evidências, tais como: efeito da idade, do desempenho intelectual; correlações com outros instrumentos validados de atenção e FE e; comparação do desempenho entre amostras clínicas com queixas e diagnósticos que indicam prejuízos na atenção e FE.

\section{Referências}

Benton, A. L., \& Hamsher, K. D. (1989). Multilingual Aphasia Examination. Iowa City, IA: AJA Associates.

Capovilla, A. G. S., \& Dias, N. M. (2008). Desenvolvimento de habilidades atencionais em estudantes da $1^{\mathrm{a}}$ à $4^{\mathrm{a}}$ série do ensino fundamental e relação com rendimento escolar. Revista Psicopedagogia, 25(78), 198-211.

Carreiro, L. R. R., \& Teixeira, M. C. T. V. (2012). Avaliação da Atenção. In C. S. Hutz (Ed.), Avanços em avaliação psicológica e neuropsicológica de crianças e adolescentes II (Vol. 1, pp. 57-92). São Paulo, SP: Casa do Psicólogo.

Carvalho, L. F. (2012). Considerações sobre avaliação psicológica e psicometria no uso de instrumentos neuropsicológicos. In A. G. Seabra \& N. M. Dias (Eds.), Avaliação neuropsicológica cognitiva: Atenção e funções executivas (Vol. 1, pp. 28-32). São Paulo, SP: Memnon.

Charchat-Fichman, H., \& Oliveira, R. M. (2009). Performance of 119 Brazilian children on Stroop paradigm: Victoria version. Arquivos de Neuro-Psiquiatria, 67(2b), 445-449. doi:10.1590/ S0004-282X2009000300014

Dias, N. M. (2009). Avaliação neuropsicológica das funções executivas: Tendências desenvolvimentais e evidências de validade de instrumentos (Dissertação de mestrado, Universidade Presbiteriana Mackenzie, São Paulo, SP, Brasil). 
Dias, N. M., \& Seabra, A. G. (2012). Executive demands of the Tower of London task in Brazilian teenagers. Psychology \& Neuroscience, 5(1), 63-75. doi:10.3922/j.psns.2012.1.09

Dias, N. M., \& Seabra, A. G. (2014). The FAS fluency test in Brazilian children and teenagers: Executive demands and the effects of age and gender. Arquivos de Neuro-Psiquiatria, 72(1), 55-62. doi:10.1590/0004-282X20130213

Duncan, M. T. (2006). Obtenção de dados normativos para desempenho no teste de Stroop num grupo de estudantes do ensino fundamental em Niterói. Jornal Brasileiro de Psiquiatria, 55(1), 42-48. doi:10.1590/S0047-20852006000100006

Hamdan, A. C., Pereira, A. P. A., \& Riechi, T. I. J. S. (2011). Avaliação e reabilitação neuropsicológica: Desenvolvimento histórico e perspectivas atuais [Número especial]. Interação em Psicologia, 15, 47-58. doi:10.5380/psi.v15i0.25373

Hazin, I., Falcão, J. T. R., Garcia, D., Gomes, E., Cortez, R., Maranhão, S., ...Dias, M. G. B. B. (2012). Dados normativos do Teste de Atenção por Cancelamento (TAC) em estudantes do ensino fundamental. Psico (Porto Alegre), 43(4), 428-436.

Krikorian, R., Bartok, J., \& Gay, N. (1994). Tower of London procedure: A standard method and developmental data. Journal of Clinical and Experimental Neuropsychology, 16, 40-50. doi:10.1080/01688639408402697

León, C. B. R., Rodrigues, C. C., Seabra, A. G., \& Dias, N. M. (2013). Funções executivas e desempenho escolar em crianças de 6 a 9 anos de idade. Revista Psicopedagogia. 30(92), 113 120.

Lezak, M. D., Howieson, D. B., \& Loring, D. W. (2004). Neuropsychological assessment. New York: Oxford University Press.

Lima, R. F., Azoni, C. A. S., \& Ciasca, S. M. (2011). Attentional performance and executive functions in children with learning difficulties. Psicologia: Reflexão e Crítica, 24(4), 685-691. doi:10.1590/S0102-79722011000400008

Lima, R. F., Tabaquim, M. L. M., \& Ciasca, S. M. (2010). Sistema atencional e funções executivas na infância. In S. M. Ciasca (Ed.), TDAH: Transtorno de Déficit de Atenção e Hiperatividade (Vol. 1, pp. 1-22). Rio de Janeiro, RJ: Revinter.
Lima, R. F., Travaini, P. P., \& Ciasca, S. M. (2009). Amostra de desempenho de estudantes do ensino fundamental em testes de atenção e funções executivas. Psicopedagogia, 26(80), 188-199.

Malloy-Diniz, L. F., Paula, J. J., Loschiavo-Alvares, F. Q., Fuentes, D., \& Leite, W. B. (2010). Exame das funções executivas. In L. F. Malloy-Diniz, D. Fuentes, P. Mattos, \& N. Abreu (Eds.), Avaliação neuropsicológica (pp. 94-113). Porto Alegre, RS: Artmed.

Menezes, A., Godoy, S., Teixeira, L. R. R. C., Carreiro, L. R. R., \& Seabra, A. G. (2012). Definições teóricas acerca das funções executivas e da atenção. In A. G. Seabra \& N. M. Dias (Eds.), Avaliação neuropsicológica cognitiva: Atenção e funções executivas (Vol. 1, pp. 34-41). São Paulo, SP: Memnon.

Pereira, A. P. P., Seabra, A. G., Dias, N. M., Trevisan, B. T., \& Prado, J. M. (2011). Funções executivas em crianças pré-escolares: Desenvolvimento da atenção seletiva medida pelo Teste de Atenção por Cancelamento. Cadernos de Psicopedagogia, 25(78).

Regard, M. (1981). Cognitive rigidity and flexibility: A neuropsychological study (Ùnpublished doctoral dissertation). University of Victoria, British Columbia, Victoria, Canada.

Reitan, R. M. (1992). Trail making test: Manual for administration and scoring. Tucson, AZ: Reitan Neuropsychology Laboratory.

Seabra, A. G., \& Dias, N. M. (2010). Habilidades atencionais: Estudo de validade de instrumentos em estudantes do ensino fundamental II. Avaliação Psicológica, 9(2), 187-198.

Simão, A. N. P., Lima, R. F., Natalin, J. C., \& Ciasca, S. M. (2010). Comparação do desempenho de estudantes em instrumentos da atenção e funções executivas. Revista Psicopedagogia, 27(83), 171-80.

Spreen, O., \& Strauss, E. (1998). A compendium of neuropsychological test-administration, norms and commentary. New York: Oxford University Press.

Stein, L. M. (1994). Teste de Desempenho Escolar: Manual para aplicação e interpretação. São Paulo, SP: Casa do Psicólogo.

Tunstall, J. R. (1999). Improving the utility of Tower of London: a neuropsychological test of plan- 
ning (Doctoral dissertation). Faculty of Healthy Sciences, Griffith University, Queensland, Australia.

Urbina, U. (2007). Fundamentos da testagem psicológica. Porto Alegre, RS: Artes Médicas.

Wechsler, S. (2003). DFH-III: O desenho da figura humana: Avaliação do desenvolvimento cognitivo de crianças brasileiras. Campinas, SP: Editora da Pontifícia Universidade Católica de São Paulo.
Weintraub, S., \& Mesulam, M. M. (1985). Mental status assessment of young and elderly adults in behavioral neurology. In M. M. Mesulam (Ed.), Principles of behavioral neurology (pp. 101103). Philadelphia, PA: FA Davis.

Recebido: $16 / 04 / 2014$

$1^{a}$ revisão: 16/07/2014 Aceite final: 24/09/2014 